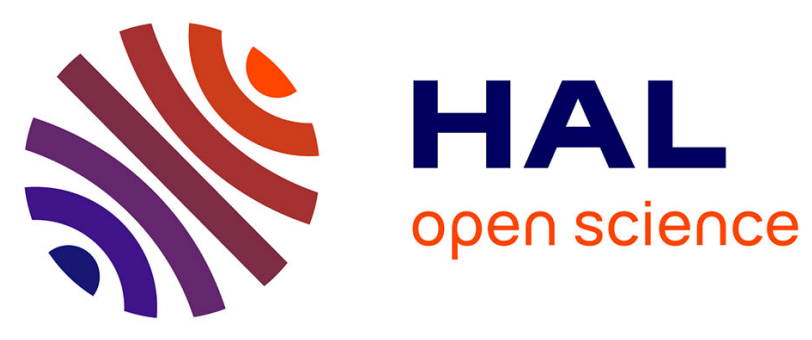

\title{
Predictive criteria of response to endoscopic treatment for severe strictures in primary sclerosing cholangitis
}

Nora Cazzagon, Olivier Chazouillères, Christophe Corpechot, Sanaâ El

Mouhadi, Edouard Chambenois, Benoit Desaint, Ulriikka Chaput, Sara

Lemoinne, Lionel Arrivé

\section{To cite this version:}

Nora Cazzagon, Olivier Chazouillères, Christophe Corpechot, Sanaâ El Mouhadi, Edouard Chambenois, et al.. Predictive criteria of response to endoscopic treatment for severe strictures in primary sclerosing cholangitis. Clinics and Research in Hepatology and Gastroenterology, 2019, 43 (4), pp.387394. 10.1016/j.clinre.2019.01.003 . hal-02291718

\section{HAL Id: hal-02291718 \\ https: / hal.sorbonne-universite.fr/hal-02291718}

Submitted on 19 Sep 2019

HAL is a multi-disciplinary open access archive for the deposit and dissemination of scientific research documents, whether they are published or not. The documents may come from teaching and research institutions in France or abroad, or from public or private research centers.
L'archive ouverte pluridisciplinaire HAL, est destinée au dépôt et à la diffusion de documents scientifiques de niveau recherche, publiés ou non, émanant des établissements d'enseignement et de recherche français ou étrangers, des laboratoires publics ou privés. 
Predictive criteria of response to endoscopic treatment for severe strictures in primary sclerosing cholangitis.

Nora Cazzagon ${ }^{1,2}$, Olivier Chazouillères ${ }^{1}$, Christophe Corpechot ${ }^{1}$, Sanaâ El Mouhadi ${ }^{3}$, Edouard Chambenois $^{3}$, Benoit Desaint ${ }^{1,4}$, Ulriikka Chaput ${ }^{1,4}$, Sara Lemoinne ${ }^{1}$, Lionel Arrivé ${ }^{3}$

${ }^{1}$ Reference Center for Inflammatory Biliary Disease and Autoimmune Hepatitis (MIVB), Saint-Antoine Hospital, Assistance Publique-Hôpitaux de Paris, APHP, Paris, France.

2 Department of Surgery, Oncology and Gastroenterology (DiSCOG), University of Padova, Padova, Italy.

${ }^{3}$ Department of Radiology, Saint-Antoine Hospital, Assistance Publique-Hôpitaux de Paris APHP, Sorbonne University, Paris, France.

${ }^{4}$ Endoscopic Department, Saint-Antoine Hospital, Assistance Publique-Hôpitaux de Paris, APHP, Paris, France

\section{Corresponding author}

Prof. Lionel Arrivé

Department of Radiology - Saint-Antoine Hospital

184 Rue du Faubourg Saint-Antoine - 75012 Paris

Telephone: 33(1) 49282258 Fax: 33(1) 49282259

E-mail: lionel.arrive@aphp.fr 


\section{ABSTRACT}

Background The aim of this study was to identify predictive criteria of improvement after endoscopic treatment (ET) for severe strictures of extrahepatic bile ducts in patients with primary sclerosing cholangitis (PSC).

Methods PSC patients who had at least one ET for severe stricture were included. Features of magnetic resonance cholangiography $(M R C)$, performed before $E T$, were evaluated according to a standard model of interpretation, and a radiologic qualitative score of probability of improvement after ET was built. Score 3 (likely) was given in case of severe common bile duct (CBD) stricture with marked dilatation without severe strictures of upstream ducts, score 1 (unlikely) was given in case of severe multiple strictures of secondary ducts without biliary dilatation and score 2 (undeterminate) was given to an intermediate pattern. The response to ET was assessed at 2 months (T2-response) from the last ET and at 12 months (T12-response) from inclusion.

Results Thirty-one patients were included. All had severe stricture (reduction $\geq 75 \%$ of the diameter) of $\mathrm{CBD}$ and $50 \%$ had severe stricture of right and/or left hepatic duct (LHD) at MRC before ET. According to the qualitative score, 16 patients had score 3, 7 had score 1 and 9 had score 2. T12-response was obtained in $50 \%$ of patients. In univariate analysis, short LHD strictures, bilirubin, transaminases, pruritus and score 3 were associated with T12response. Increased bilirubin and transaminases were independent predictive factors of T12response (HR 24, 95\% Cl:3.4-170.4, $\mathrm{p}=0.001$ and 23.8, 95\%Cl3.4-169.4, $\mathrm{p}=0.002$, respectively).

Conclusion MRC, together with biochemical features, may contribute to identify the PSC patients who are likely to be improved after ET for severe strictures of extrahepatic bile ducts. 


\section{KEYWORDS}

Dominant stenosis, endoscopic retrograde cholangiopancreatography, magnetic resonance imaging, sensitivity and specificity

\section{ABBREVIATIONS}

PSC, Primary Sclerosing Cholangitis

ERCP, Endoscopic Retrograde Cholangiopancreatography

CBD, Common Bile Duct

RHD, Right Hepatic Duct

LHD, Left Hepatic Duct

ET, Endoscopic Treatment

MRC, Magnetic Resonance Cholangiography

IHBD, Intrahepatic Bile Duct

EHBD, Extrahepatic Bile Duct 


\section{INTRODUCTION}

Primary sclerosing cholangitis (PSC) is a chronic cholestatic liver disease characterized by progressive fibrotic obliteration of intra- and/or extrahepatic bile ducts [1]. PSC patients can experience symptoms such as pruritus and abdominal pain or suffer from acute cholangitis and jaundice. These clinical manifestations are frequently related to the development of dominant strictures. The term "dominant stricture" has been defined by a stricture displaying a diameter of $\leq 1.5 \mathrm{~mm}$ when affecting common bile duct (CBD) or a diameter of $\leq$ $1.0 \mathrm{~mm}$ when affecting right (RHD) or left hepatic duct (LHD), within $2 \mathrm{~cm}$ after bifurcation, assessed during endoscopic retrograde cholangiopancreatography (ERCP) [2]. The endoscopic treatment (ET) for dominant strictures in PSC can improve clinical parameters [2-7], liver function tests $[2-10]$ and cholangiogram $[5,6,11]$. Moreover, some results suggested that ET for dominant stricture could also improve survival, based on comparison of observed survival with the survival predicted by the Mayo risk score $[2,9,12]$. However, in PSC patients, cholestatic enzymes may spontaneously fluctuate, both in patients with and without dominant strictures, demonstrating that the presence of a dominant stricture is not necessary the cause of increased cholestatic liver enzymes [13]. Moreover, the presence of severe stricture in the extrahepatic bile ducts was not found to be associated to radiologic progression in PSC patients over a mean follow-up of 4 years [14]. The occurrence of complications after ERCP with or without ET ranges from 4 to $18 \%$ in PSC patients [1], with an increased incidence of post-ERCP pancreatitis compared with patients without PSC [15]. Taken together, all these evidences underline the need to be able to identify patients who could benefit from ET for dominant stricture. Magnetic resonance cholangiography (MRC) had substituted ERCP as first choice modality for diagnosing PSC, as a result of its accuracy 
and non-invasiveness. MRC is also recommended prior to ERCP in PSC patients displaying a rapid cholestatic impairment and/or new symptoms [16]. Compared to ERCP, MRC does not induce hydrostatic pressure and has a lower spatial resolution to visualize extrahepatic bile ducts; therefore the definition of "dominant stricture" cannot be applied in MRC. The term of "severe stricture" has been proposed for strictures of extrahepatic bile duct displaying a decrease of at least $75 \%$ in the diameter of the original duct on MRC [14]. To date, no study has explored whether some MRC features could be used to define strictures that require ET and thus to identify patients who could improve after ET. In clinical practice, a risk-to-benefit assessment is recommended before performing ERCP in PSC patient[16]. For instance, in case of stable severe stricture with or without acute clinical manifestations (acute cholangitis or jaundice), the decision to perform ERCP with ET is not clear-cut, because of a lack of predictive criteria of response to ET. Therefore, the aim of this preliminary study was to investigate the existence of radiological and clinical predictive criteria of short-term improvement after ET in PSC patients that could be subsequently validated in a multicentre study.

\section{PATIENTS AND METHODS}

We included large-duct PSC patients who underwent at least one ET for dominant stricture in our centre between 2005 and February 2017. Inclusion criteria were performance of a MRC within 3 months before ET and at least 1 year of follow-up after the first ET. The date of inclusion was defined by the date of first ET. Exclusion criteria were coexisting liver diseases (except for overlap syndrome with autoimmune hepatitis that were included), recurrence of PSC after liver transplantation, decompensated cirrhosis at inclusion, 
biliodigestive anastomosis, previous or current diagnosis of cholangiocarcinoma or diagnosis of cholangiocarcinoma within 6 months after the inclusion.

Our faculty hospital's Institutional Review Board approved the review of radiological and clinical data for this study. Informed consent was waived for this retrospective analysis of data.

\section{MRC technique}

MRC was performed according to the protocol for 3D-MRC, in line with the recommendations of International PSC study group [17]. Three radiologists (two senior radiologists, LA (radiologist 1) and SEM (radiologist 2), with 20 and 10 years of experience in MRC and a junior radiologist, EC (radiologist 3), with 2 years of experience, blinded to clinical data, analysed in consensus the MRC performed before ET, by using a previously described standard model of interpretation [14]. This model includes analysis of the following features: intrahepatic (IHBD) and extrahepatic bile duct (EHBD) with regard to strictures, dilatations, and biliary wall enhancement after contrast injection, presence of gallstones as well as associated liver-related signs including dysmorphy, heterogeneity of liver enhancement after gadolinium-based contrast agents injection, and portal hypertension. According to this model, a severe stricture of EHBD is defined by a decrease of at least $75 \%$ in the diameter of the original duct. Dysmorphy is defined by a significant atrophy of either right or left hepatic lobe and/or presence of marked lobulations of liver surface and/or an increased caudate/right lobe ratio. Portal hypertension is defined by presence of portosystemic shunts with or without splenomegaly [14]. Each MRC was also reviewed independently by each of the three radiologists, according to a qualitative score (proposed by radiologist 1 ). This qualitative score aimed to evaluate the probability of improvement after ET. The score was 
classified as follows: score 3 (improvement likely) was given in case of severe CBD stricture with marked upstream biliary dilatation without severe stricture of upstream biliary ducts (Figure 1A); score 1 (improvement unlikely) was given in case of multiple severe strictures of CBD, RHD and LHD and IHBD without significant upstream biliary dilatation (Figure 1B). Score 2 (improvement undeterminate), was given to an intermediate pattern (Figure 1C). Each MRC was revaluated twice, 6 weeks apart, by radiologist 1 in order to assess intraobserver variability.

\section{Clinical data}

Clinical and biochemical data at inclusion (time of first ET), at 2 months after the last ET (T2) and at 12 months after the inclusion (T12) were collected by reviewing medical records. Details regarding the type of ET (simple balloon dilatation or balloon dilatation associated to short-term biliary stenting) were collected for each procedure performed during the period of observation. Failure of the procedure was defined when ET (balloon dilatation +/- stent placement) proved impossible after 2 ERCPs.

The response to ET was assessed in patients without failure of the procedure, at T2 and T12 and was defined by either the presence of at least one clinical criterion (resolution of jaundice or acute bacterial cholangitis, resolution or decrease of right upper abdominal pain or pruritus) or the presence of the biochemical criterion (simultaneous decrease of at least $40 \%$ from baseline values in total bilirubin, alkaline phosphatase and GGT levels).

\section{Statistical analysis}

Continuous variables were expressed as median and interquartile ranges (IQR: 25th to the 75th percentiles). Dichotomous variables were expressed as number and percentages. Continuous variables were compared using the Wilcoxon-Mann-Whitney test. Quantitative 
variables were compared using the chi-square test or Fisher's exact test when appropriate to compare features in the two groups (responders vs. non-responders). A difference was considered significant when $p<0.05$. Univariate analysis and multivariate logistic regression analysis were performed to identify predictive factors of response to ET at the two time points. The multivariate analysis included variables found significant in the univariate analysis. Continuous variables were included after dichotomisation in binomial variables using median values as cutoff. Radiological variables were categorized in binomial variables according to the presence or absence of the most severe feature. Intraobserver variability was assessed using Cohen's k coefficient [18] and interobserver variability was evaluated using Fleiss' $\mathrm{k}$ coefficient [19]. Interpretation of the kappa values was performed according to the guidelines of Landis and Koch [20] with kappa values $>0.8$ classified as excellent agreement, 0.79 to 0.60 substantial, 0.59 to 0.40 moderate, 0.39 to 0.20 slight and $<0.20$ poor agreement.

\section{RESULTS}

\section{Study population}

We included 31 PSC patients who underwent at least one ET for dominant stricture, preceded by an MRC. All patients had PSC affecting both intra and extrahepatic bile ducts and all patients were treated with ursodeoxycholic acid (median dose of $12.5 \mathrm{mg} / \mathrm{Kg} / \mathrm{day}$ ). Patients' clinical and biochemical characteristics are reported in Table 1. Half of patients $(n=15)$ had acute cholangitis or jaundice at inclusion. 


\section{Magnetic resonance imaging findings}

The mean interval time between MRC and ERCP was $79 \pm 71$ days. Magnetic resonance imaging findings are reported in Table 2. All patients had a severe CBD stricture that was associated to an upstream dilatation in $20 \%$ of patients. Moreover, $71 \%$ and $54 \%$ of patients had a severe stricture in RHD and LHD. All patients had strictures affecting more than $25 \%$ of IHBD, severe IHBD strictures were present in all but one patient, and the majority of patients had moderate to marked IHBD dilatation. Eight patients (26\%) displayed intraductal stones. Dysmorphy and signs of portal hypertension were observed in $74 \%$ and $29 \%$ of patients, respectively. IHBD or EHBD enhancement after gadolinium-based contrast agent injection was present in around $40 \%$ of patients.

According to the qualitative score of improvement evaluated by radiologist 1 at first evaluation, seven patients were judged unlikely to improve after ET (score 1), 16 patients were judged likely to improve after ET (score 3) and finally, nine patients had an

undeterminate pattern of improvement (score 2). Intraobserver variability between repeated evaluations by radiologist 1 was $74 \%, k=0.6$ indicating substantial agreement. Interobserver variability of the qualitative score of improvement between the three radiologists was $60 \%, \mathrm{k}=0.40$, indicating fair agreement.

\section{ERCP findings and endoscopic treatment}

According to our local policy, all PSC patients followed-up in our centre were monitored by annual MRC. Moreover, in case of clinical or biochemical aggravation, a new MRC was performed. The decision to perform an ERCP with ET, made by a multidisciplinary staff, was based on the presence of a severe stricture in the EHBD found at MRC, associated to radiologic progression, clinical or biochemical worsening. Multidisciplinary staff evaluated the risk-benefit ratio of ET, on a case by case basis. All the patients showed the presence of 
at least one dominant stricture in $\mathrm{CBD}$ at ERCP. A single dominant stricture localized in CBD was observed in 17 patients, otherwise dominant stricture in both CBD and RHD were found in three patients and finally, seven patients had multiple dominant strictures localized in $\mathrm{CBD}, \mathrm{RHD}$ and LHD. Four patients had dominant stricture in $\mathrm{CBD}$ associated to the presence of intraductal stones. The majority of patients underwent sphincterotomy with balloon dilatation and stent placement. Technical success of ET was obtained in 27 patients at first ERCP, in three patients at second ERCP and a technical failure (failure to pass the stricture) was documented in one patient. Most of patients needed a second ERCP within a median time of 41 days from the first procedure to complete the dilatation or to remove the biliary stent. Brush cytology was obtained in each patient without initial failure and presence of neoplastic cells in the cytologic sample was excluded in all cases. The subsequent clinical and radiological follow-up confirmed the benign nature of the dominant stricture.

Seven post-procedure complications (11\%) were reported in seven patients (22\%). Three patients developed post-ERCP acute pancreatitis, two patients had post sphincterotomy gastrointestinal bleeding, one patient developed biliary peritonitis with subsequent cholecystectomy and one patient had a post-ERCP duodenal perforation. A history of one or more acute cholangitis after the first ERCP was observed in 19 patients, of them, one was transplanted within 12 months from first ERCP for recurrent acute cholangitis and one patient developed cirrhotic decompensation.

\section{Evaluation of response to ET}

The response to ET was observed in 16 patients (53\%) at T2 and in 15 patients (50\%) at T12. In the total cohort, a significant improvement of total bilirubin and aspartate 
aminotransferase was observed at end of follow-up [35(17-89) vs. 16(11-28) $\mu \mathrm{mol} / \mathrm{L} p<0.01$ and 72(47-141) vs. 54(28-89) U/L, p=0.03, respectively].

Responders at T2 were characterized, at inclusion, by significantly higher total bilirubin [63(33-171) vs. 23(12-62), $p=0.03$ ], AST [105(69-211) vs. 52(39-89) $U / L, p=0.01]$ and ALP serum levels [356(208-565) vs. 203(153-271) U/L, p=0.02], lower prothrombin time [82(6694) vs. 99(89-110), $p=0.01$ ], a higher frequency of pruritus and upper right abdominal pain (63\% vs. $8 \%, p<0.01$ and $44 \%$ vs. $0 \%, p=0.01$, respectively), a higher frequency of severe $(\geq 9$ $\mathrm{mm}$ ) LHD dilatations and short $(\leq 10 \mathrm{~mm}$ ) LHD stricture $(44 \%$ vs. $8 \%, \mathrm{p}=0.04$ and $94 \%$ vs. $54 \%, p=0.03$, respectively) compared to $\mathrm{T} 2$ non-responders. In univariate analysis, variables significantly associated with response to ET at T2 were the presence of pruritus, total bilirubin, AST, ALP, the presence of short LHD strictures and severe LHD dilatation (data not shown). Independent predictors of response at $\mathrm{T} 2$, identified by multivariate analysis, were the presence of short LHD stricture (HR 24, 95\% Cl 1.3-464.8, $\mathrm{p}=0.03$ ) and AST (HR 27, 95\%Cl $2-362, p=0.01)$.

Responders at T12 were characterized, at inclusion, by higher AST serum level (113(72-225) vs. 53(41-68), $p<0.01)$, higher bilirubin (75(51-172) vs. 20(12-30), $p=0.02)$, lower PT (87(65$99)$ vs. $98(89-109), p=0.03)$ higher frequency of pruritus ( $67 \%$ vs. $7 \%, p<0.01)$ and severe CBD dilatations ( $27 \%$ vs. $0, p=0.05)$, short $(\leq 10 \mathrm{~mm})$ LHD strictures ( $93 \%$ vs. $57 \%, p=0.04)$ and qualitative score $3(67 \%$ vs. $29 \%, p=0.05)$. In univariate analysis, total bilirubin, AST, presence of pruritus, short LHD strictures and qualitative score 3 (Figure 2) were associated to response at T12 (data not shown). In multivariate analysis, total bilirubin and AST at inclusion were independent predictive factors of response at T12 (HR 19.1, 95\% Cl: 1.7-221.8, $p=0.02$ and $H R$ 18.8, 95\%Cl: 1.6-222, $p=0.02)$. 


\section{DISCUSSION}

This is the first study that aims to identify MRC and clinical predictive criteria of improvement after ET in PSC patients with severe extrahepatic bile duct strictures. In a monocentric cohort of large-duct PSC patients, we assessed radiological and clinical features before ET for severe stricture. We used a standard model of MRC interpretation of PSC features [14] and we found that, at inclusion, a severe stricture of $C B D$, defined by a decrease of at least $75 \%$ in the diameter of the original duct, was present in all treated patients. Moreover, half of the patients had also a severe stricture of RHD and/or LHD.

We evaluated the difference between responders and non-responders and we observed that 12-months responders were characterized, at inclusion, by a more symptomatic disease, by a higher frequency of severe CBD dilatation, short LHD strictures and qualitative score 3. However, total bilirubin and transaminases were the only two independent predictors of response to ET in multivariate analysis. Thus, we observed that a better response to ET was achieved in PSC patients having acute clinical manifestation and probably in patients with severe dilatation of the CBD and short stricture of the LHD. However, further conclusions cannot be drawn from our present results, because of the low number of included patients that limited the statistical power of the study.

Recently, Zenouzi et al. described a number of relevant points regarding the real-life interpretation of MRC in PSC patients and reported some data which differ from our results [21]. First, interobserver agreement between experts regarding the decision to perform ET in case of significant strictures was poor $(k=0.12)$ [21] and was lower than the interobserver agreement of the qualitative score of improvement reported in our study $(k=0.40)$. One of the explanations for this discrepancy could be the fact that Zenouzi's study involved different specialists with variable expertise (hepatologist, gastroenterologist and radiologist) 
[21] whereas ours involved only radiologists. Moreover, Zenouzi et al. showed that a high level of total bilirubin and the presence of acute cholangitis were the main reasons to indicate ET. Differently, in our cohort, only half of patients had jaundice or acute cholangitis. Finally, Zenouzi et al. reported the absence of concordance between a priori probability of improvement after ET and the observed results of the procedure [21]. Taken together, these data confirm the need to have objective predictive criteria of improvement after ET, especially in patients with severe stricture but without acute clinical manifestation (jaundice or acute cholangitis). In fact, short-term improvement of symptoms and biochemistry after ET was recently reported in $77 \%$ of PSC patients having a dominant stricture without acute cholangitis [22]. Moreover, results of the DILSTENT trial showed that balloon dilatation and short-term stenting had similar efficacy in term of recurrence-free survival, improvement of symptoms and cholestatic enzymes [22].

In the current study, we defined an arbitrary criterion of response to ET that assessed clinical and biochemical, but not radiological changes, similarly to what has been previously proposed [23]. Here, the biochemical criterion was defined as a simultaneous improvement of three liver enzymes (total bilirubin, GGT, ALP) in order to reduce the probability that a spontaneous fluctuation of one or two liver enzymes could be interpreted as a biochemical response.

One limitation of our study is the rather short follow-up (12 months) letting unresolved the question of the long-term efficacy of ET, notably in term of transplant-free survival. Another limitation, that we could overcome by expanding the study cohort, is the lack of adjustment for different factors: indication to ET, technical failure at first ERCP, complications, age, number of ET, number and localization of strictures (only in the CBD vs. in the CBD + RHD or LHD) and type of endoscopic treatment (simple balloon dilatation vs. short-term stenting). 
We reported an initial technical failure of ET in $3.2 \%$ patients, this rate is comparable to the one recently reported in the DILSTENT trial (5\%) [22]. Finally, in our study, moderate to severe complications developed after $11 \%$ of procedures, similarly to previously described [1], confirming that a risk-to-benefit evaluation is mandatory before performing ET and that non-invasive predictive criteria of response are clearly needed.

In conclusion, in PSC patients with severe strictures of extrahepatic bile ducts, MRC together with biochemical features may contribute to identify patients who are likely to be improved after ET. A validation in a larger cohort is warranted to confirm these results, especially in patients without an evident indication to ET.

\section{REFERENCES}

[1] Karlsen TH, Folseraas T, Thorburn D, Vesterhus M. Primary sclerosing cholangitis - a comprehensive review. J Hepatol 2017;67:1298-323. doi:10.1016/j.jhep.2017.07.022.

[2] Stiehl A, Rudolph G, Klöters-Plachky P, Sauer P, Walker S. Development of dominant bile duct stenoses in patients with primary sclerosing cholangitis treated with ursodeoxycholic acid: outcome after endoscopic treatment. J Hepatol 2002;36:151-6.

[3] Kaya M, Petersen BT, Angulo P, Baron TH, Andrews JC, Gostout CJ, et al. Balloon dilation compared to stenting of dominant strictures in primary sclerosing cholangitis. Am J Gastroenterol 2001;96:1059-66. doi:10.1111/j.1572-0241.2001.03690.x.

[4] Johnson GK, Geenen JE, Venu RP, Hogan WJ. Endoscopic treatment of biliary duct strictures in sclerosing cholangitis: follow-up assessment of a new therapeutic approach. Gastrointest Endosc 1987;33:9-12.

[5] Johnson GK, Geenen JE, Venu RP, Schmalz MJ, Hogan WJ. Endoscopic treatment of biliary tract strictures in sclerosing cholangitis: a larger series and recommendations for treatment. Gastrointest Endosc 1991;37:38-43.

[6] Lee JG, Schutz SM, England RE, Leung JW, Cotton PB. Endoscopic therapy of sclerosing cholangitis. Hepatology 1995;21:661-7.

[7] Ponsioen CY, Lam K, van Milligen de Wit AW, Huibregtse K, Tytgat GN. Four years experience with short term stenting in primary sclerosing cholangitis. Am J Gastroenterol 1999;94:2403-7. doi:10.1111/j.1572-0241.1999.01364.x.

[8] Stiehl A, Rudolph G, Sauer P, Benz C, Stremmel W, Walker S, et al. Efficacy of ursodeoxycholic acid treatment and endoscopic dilation of major duct stenoses in primary 
sclerosing cholangitis. An 8-year prospective study. J Hepatol 1997;26:560-6.

[9] Baluyut AR, Sherman S, Lehman GA, Hoen H, Chalasani N. Impact of endoscopic therapy on the survival of patients with primary sclerosing cholangitis. Gastrointest Endosc 2001;53:308-12.

[10] Gotthardt DN, Rudolph G, Klöters-Plachky P, Kulaksiz H, Stiehl A. Endoscopic dilation of dominant stenoses in primary sclerosing cholangitis: outcome after long-term treatment. Gastrointest Endosc 2010;71:527-34. doi:10.1016/j.gie.2009.10.041.

[11] Wagner S, Gebel M, Meier P, Trautwein C, Bleck J, Nashan B, et al. Endoscopic management of biliary tract strictures in primary sclerosing cholangitis. Endoscopy 1996;28:546-51. doi:10.1055/s-2007-1005552.

[12] Gluck M, Cantone NR, Brandabur JJ, Patterson DJ, Bredfeldt JE, Kozarek RA. A twentyyear experience with endoscopic therapy for symptomatic primary sclerosing cholangitis. J Clin Gastroenterol 2008;42:1032-9. doi:10.1097/MCG.0b013e3181646713.

[13] Björnsson E, Lindqvist-Ottosson J, Asztely M, Olsson R. Dominant strictures in patients with primary sclerosing cholangitis. Am J Gastroenterol 2004;99:502-8. doi:10.1111/j.1572-0241.2004.04106.x.

[14] Ruiz A, Lemoinne S, Carrat F, Corpechot C, Chazouillères O, Arrivé L. Radiologic course of primary sclerosing cholangitis: assessment by three-dimensional magnetic resonance cholangiography and predictive features of progression. Hepatology 2014;59:242-50. doi:10.1002/hep.26620.

[15] von Seth E, Arnelo U, Enochsson L, Bergquist A. Primary sclerosing cholangitis increases the risk for pancreatitis after endoscopic retrograde cholangiopancreatography. Liver Int 2015;35:254-62. doi:10.1111/liv.12640.

[16] Aabakken L, Karlsen TH, Albert J, Arvanitakis M, Chazouilleres O, Dumonceau J-M, et al. Role of endoscopy in primary sclerosing cholangitis: European Society of Gastrointestinal Endoscopy (ESGE) and European Association for the Study of the Liver (EASL) Clinical Guideline. Endoscopy 2017;49:588-608. doi:10.1055/s-0043-107029.

[17] Schramm C, Eaton J, Ringe KI, Venkatesh S, Yamamura J, MRI working group of the IPSCSG. Recommendations on the use of magnetic resonance imaging in PSC-A position statement from the International PSC Study Group. Hepatology 2017;66:1675-88. doi:10.1002/hep.29293.

[18] Cohen J. A Coefficient of Agreement for Nominal Scales. Educational and Psychological Measurement 1960;20:37-46. doi:10.1177/001316446002000104.

[19] Fleiss JL. Measuring nominal scale agreement among many raters. Psychological Bulletin 1971;76:378-82. doi:10.1037/h0031619.

[20] Landis JR, Koch GG. The Measurement of Observer Agreement for Categorical Data. Biometrics 1977;33:159-74. doi:10.2307/2529310.

[21] Zenouzi R, Liwinski T, Yamamura J, Weiler-Normann C, Sebode M, Keller S, et al. Follow-up magnetic resonance imaging/3D-magnetic resonance cholangiopancreatography 
in patients with primary sclerosing cholangitis: challenging for experts to interpret. Aliment Pharmacol Ther 2018;48:169-78. doi:10.1111/apt.14797.

[22] Ponsioen $\mathrm{CY}$, Arnelo U, Bergquist A, Rauws EA, Paulsen V, Cantú P, et al. No Superiority of Stents vs Balloon Dilatation for Dominant Strictures in Patients With Primary Sclerosing Cholangitis. Gastroenterology 2018. doi:10.1053/j.gastro.2018.05.034.

[23] Enns R, Eloubeidi MA, Mergener K, Jowell PS, Branch MS, Baillie J. Predictors of successful clinical and laboratory outcomes in patients with primary sclerosing cholangitis undergoing endoscopic retrograde cholangiopancreatography. Can J Gastroenterol 2003;17:243-8.

\section{Acknowledgements}

Nora Cazzagon was granted by EASL Sheila Sherlock fellowship 2017 and 2018 for the project

"Prognostic role of magnetic resonance imaging in Primary Sclerosing Cholangitis".

This work was funded by "Fond CSP". 


\section{FIGURE LEGEND}

Figure 1. Example of qualitative score 3 (improvement likely) (Fig. 1A), score 1

(improvement unlikely) (Fig. 1B), score 2 (improvement undeterminate) (Fig.1C).

Three-dimensional T2 MRC images showing examples of the different scores of improvements. In Fig.1A image shows the presence of a severe strictures of lower part of CBD associated to a marked dilatation of upstream CBD, RHD and LHD and intrahepatic biliary ducts characteristic of score 3 (improvement after endoscopic treatment likely). In Fig. 1B the presence of multiple severe strictures of CBD, RHD and LHD and intrahepatic biliary ducts and the lack of significant upstream dilatation define the score 1 (improvement after endoscopic treatment unlikely). Fig. $\mathbf{1 C}$ demonstrates the presence of severe strictures of $C B D, R H D$ and LHD, intrahepatic biliary ducts with the presence of segments of dilated intrahepatic biliary ducts define the score 2 (improvement undeterminate).

Figure2. Distribution of responders and non-responder to endoscopic treatment at 2 months (T2) (Fig.2A) and at 12 months (T12) after endoscopic treatment (Fig.2B) according the qualitative score of improvement at baseline. 


\section{TABLES}

Table 1. Patients' characteristics at inclusion

\section{Characteristics}

Male gender, $\mathrm{n}(\%)$

20(65)

Age at inclusion

$36(27-52)$

Cirrhosis

$10(32 \%)$

Acute cholangitis

$5(16 \%)$

Jaundice

$15(48 \%)$

Pruritus

$12(39 \%)$

Right upper abdominal pain

$7(23 \%)$

Total bilirubin ( $\mu \mathrm{mol} / \mathrm{L})$

$35(17-89)$

ALP (N.R. 43-115 U/L)

262(174-379)

AST (N.R. 10-35 U/L)

72 (47-141)

GGT (N.R. 3-45 U/L)

227(94-345)

PT (N.R. 75-112\%)

91(69-100)

Abbreviations: ALP, Alkaline phosphatase, N.R., normal range, AST, aspartate aminotransferase, GGT, gamma glutamyl transpeptidase, $\mathrm{PT}$, prothrombin time 
Table 2. Radiologic findings at magnetic resonance imaging in 31 PSC patients before endoscopic treatment.

\section{MRI findings}

CBD strictures

$$
\begin{array}{cl} 
& \text { Absent } \\
\leq 75 \% \\
>75 \% \\
\text { CBD stricture length } \\
\leq 2 \mathrm{~mm} \\
3-10 \mathrm{~mm} \\
>10 \mathrm{~mm} \\
\text { CBD dilatation } \\
\leq 10 \mathrm{~mm} \\
11-14 \mathrm{~mm} \\
\geq 15 \mathrm{~mm}
\end{array}
$$

CBD enhancement

$$
\text { Absent }
$$

Thickness $<2 \mathrm{~mm}$

Thickness 2-6 mm

RHD strictures

$$
\text { Thickness }>6 \mathrm{~mm}
$$

Absent

$\leq 75 \%$

RHD stricture length

Absent

$\leq 2 \mathrm{~mm}$

3-10 mm

$>10 \mathrm{~mm}$

RHD dilatation

$\leq 6 \mathrm{~mm}$

7-8 mm

$\geq 9 \mathrm{~mm}$

RHD enhancement

Absent

Thickness $<2 \mathrm{~mm}$

Thickness 2-6 mm

Thickness $>6 \mathrm{~mm}$

LHD strictures

Absent

$\leq 75 \%$

HD stricture length

$$
\text { Absent }
$$

$\leq 2 \mathrm{~mm}$

3-10 mm

$>10 \mathrm{~mm}$

LHD dilatation

$$
\leq 6 \mathrm{~mm}
$$

7-8 mm

$\geq 9 \mathrm{~mm}$

LHD enhancement

Thickness $<2 \mathrm{~mm}$

Thickness 2-6 mm Thickness $>6 \mathrm{~mm}$

IHBD Stricture

Absent

$$
\leq 75 \%
$$

$>75 \%$

IHBD involvement

$$
\text { absent }
$$$$
>25 \%
$$

IHBD dilatation

$$
\text { None }(\leq 3 \mathrm{~mm})
$$

mild $(4 \mathrm{~mm})$

marked $(\geq 5 \mathrm{~mm}$ )

IHBD enhancement

Absent
thickness $<2 \mathrm{~mm}$

thickness 2-6 mm

thickness $>6 \mathrm{~mm}$

Intraductal stones

$$
\text { Absent }
$$

Dysmorphy

$$
\text { Present }
$$

$$
\text { Absent }
$$

Present

Portal Hypertension

$$
\begin{aligned}
& \text { Absent } \\
& \text { Present }
\end{aligned}
$$

Parenchymal enhancement heterogeneity

$$
\text { Absent }
$$

\section{PSC patients (n.31)}

$31(100 \%)$

$2(7 \%)$

$5(16 \%)$

$24(77 \%)$

$25(81 \%)$

2(6\%)

$4(13 \%)$

$14(61 \%)$

6(26\%)

$3(13 \%)$

$7(23 \%)$

2(6\%)

$22(71 \%)$

$7(23 \%)$

$3(10 \%)$

$10(32 \%)$

$11(35 \%)$

20(65\%)

$5(16 \%)$

6(19\%)

$14(61 \%)$

$6(26 \%)$

$3(13 \%)$

0

$7(23 \%)$

$7(23 \%)$

$17(54 \%)$

$7(23 \%)$

$3(10 \%)$

14(45\%)

7(23\%)

20(65\%)

$3(10 \%)$

$8(25 \%)$

$14(61 \%)$

$6(26 \%)$

$3(13 \%)$

$1(3 \%)$

$30(97 \%)$

$31(100 \%)$

$5(16 \%)$

$16(52 \%)$

$10(32 \%)$

$15(65 \%)$

$5(22 \%)$

$3(13 \%)$

$23(74 \%)$

$8(26 \%)$

$8(26 \%)$

$23(74 \%)$

$22(71 \%)$

9(29\%)

$1(4 \%)$

22(96\%) 
Abbreviations: CBD, Common bile duct; RHD, Right Hepatic Duct; LHD, Left Hepatic Duct; IHBD, Intrahepatic bile ducts 
Highlights

- In patients with primary sclerosing cholangitis, there is a need of objective predictive criteria of improvement after endoscopic treatment, especially in case of severe stricture without acute clinical manifestation (jaundice or acute cholangitis).

- Features of magnetic resonance cholangiography, performed before endoscopic treatment, were evaluated according to a standard model of interpretation, and a radiologic qualitative score of probability of improvement after endoscopic treatment was built.

- Score 3 (improvement likely) was given in case of severe common bile duct stricture with marked dilatation without severe strictures of upstream ducts, score 1 (improvement unlikely) was given in case of severe multiple strictures of secondary ducts without biliary dilatation and score 2 (undeterminate) was given to an intermediate pattern.

- Short left hepatic duct stricture, bilirubin, transaminases, pruritus and score 3 (improvement likely) were associated with response to endoscopic treatment.

- Magnetic resonance cholangiography, together with biochemical features, may contribute to identify the primary sclerosing cholangitis patients who are likely to be improved after endoscopic treatment for severe strictures of extrahepatic bile ducts.

- A validation in a larger cohort is warranted to confirm these results, especially in patients without an evident indication to endoscopic treatment. 
$1 \mathrm{~A}$

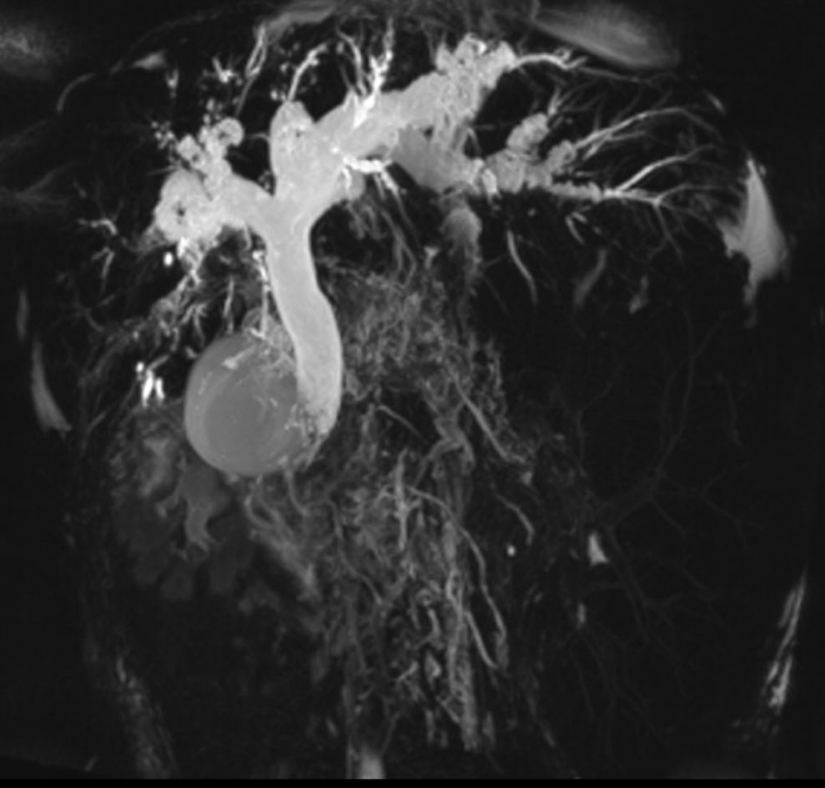

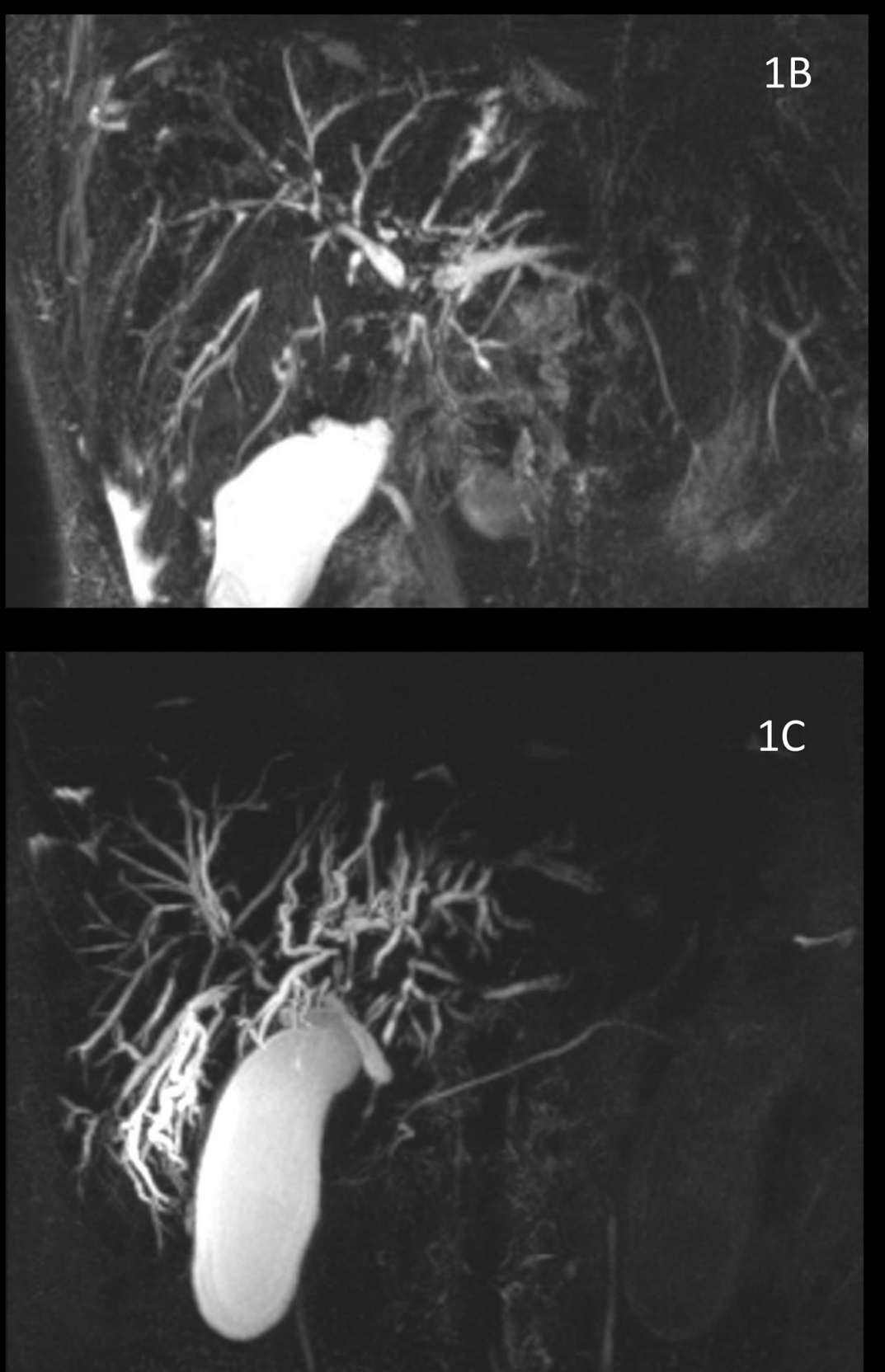


$2 A$

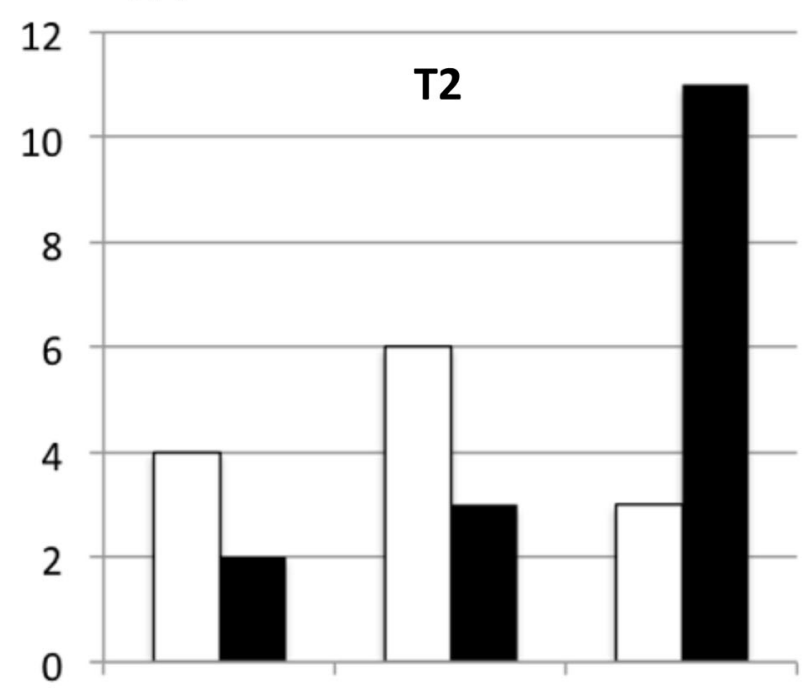

Score 1
Score 2
2B

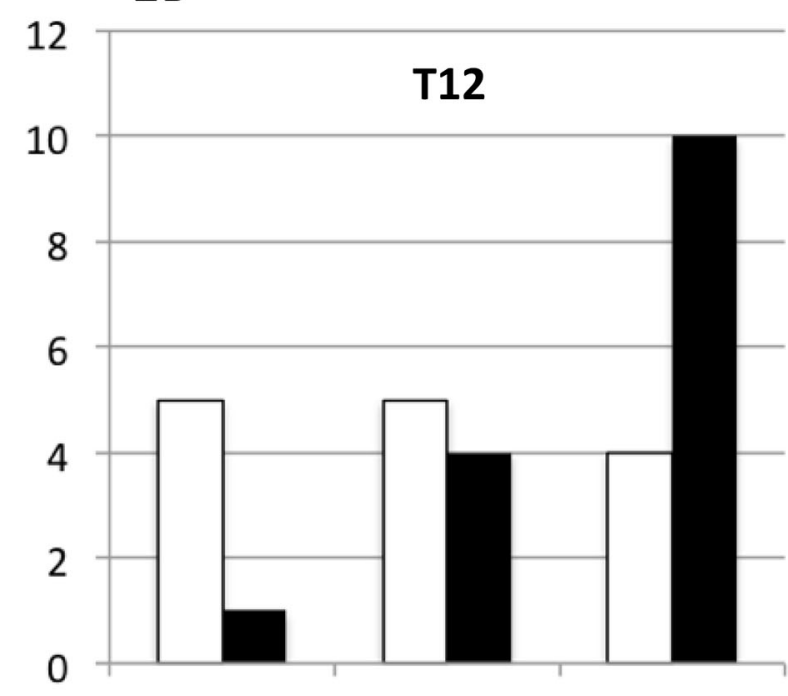

$\square$ Non-Responders

- Responders
Score 1
Score 2
Score 3 